\title{
Engineering a synthesis-friendly constitutive promoter for mammalian cell expression
}

Chee Ka Candice Lam ${ }^{1}$ and Kevin Truong ${ }^{1,2, *}$

${ }^{1}$ Institute of Biomedical Engineering, University of Toronto, 164 College Street, Toronto, Ontario, M5S 3G9, Canada

${ }^{2}$ Edward S. Rogers, Sr. Department of Electrical and Computer Engineering, University of Toronto, 10 King's College Circle, Toronto, Ontario, M5S 3G4, Canada

Lead contact $(*)$ :

Kevin Truong

kevin.truong@utoronto.ca, Tel: 416-978-7772, Fax: 416-978-4317

164 College Street Room 407, Rosebrugh Building, University of Toronto

Toronto, ON, M5S3G9, Canada 


\section{Supporting Information}

\section{Table of Contents}

Supplemental Figure 1. Synthesis-related CMVp features and stable cell lines

Supplementary Video 1. Fluorescence microscopy timelapse of HEK293 cells stably transfected with SFCp-driven CaRQ and Venus fusion construct.

Supplementary Video 2. Fluorescence microscopy timelapse of HEK293 cells stably transfected with CMVp-driven red fluorescence $\mathrm{Ca}^{2+}$ indicator, $\mathrm{RCaMP}$, in tandem with SFCpdriven Ceru-NanoLuc. 

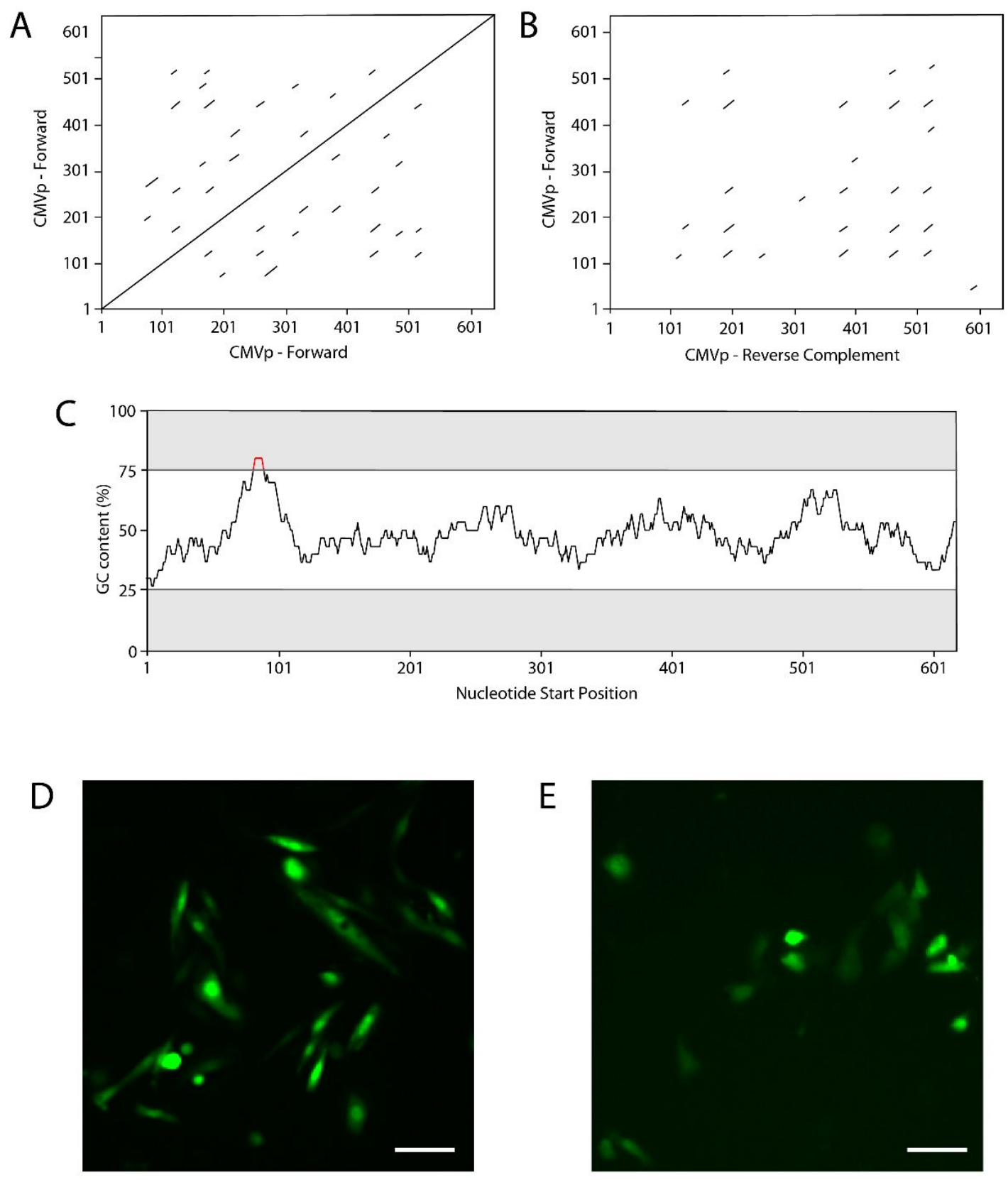

Figure S1. Synthesis-related CMVp features and stable cell lines. (A, B) Dot plots for visualization of repeating segments found in two sequences placed along the axes. Minimum window size used is 9bp. (A) Forward direction repeats present in full-length CMV promoter displayed as a dot plot with the full sequence along each axes. (B) Reverse, indirect repeats present in full-length CMV promoter displayed as a dot plot with the full sequence along the $\mathrm{Y}$ axis and reverse complement along the X-axis. (C) GC-content plot for CMVp calculated with window size of $30 \mathrm{bp}$. Red GC contents show sequences outside of the synthesis-friendly GC content limits of $25 \%$ and $75 \%$. Pseudo-coloured fluorescence microscopy images of stable CHO cells (D) and MDCK cells (E) expressing Venus regulated by SFC promotor. Scale bar represents $100 \mu \mathrm{m}$. 
Supplementary Video 1. Fluorescence microscopy timelapse of HEK293 cells stably transfected with SFCp-driven CaRQ and Venus fusion construct. Images are taken at 5 second intervals following observation period of cells to ensure absence of blebbing. Bolus 10uM ATP stimulus is added at 10 seconds. Rapid blebbing begins at 140 seconds.

Supplementary Video 2. Fluorescence microscopy timelapse of HEK293 cells stably transfected with CMVp-driven red fluorescence $\mathrm{Ca}^{2+}$ indicator, $\mathrm{RCaMP}$, in tandem with SFCpdriven Ceru-NanoLuc. Images are taken at 5 second intervals. Bolus 10uM ATP stimulus is added at 20 seconds. 